\title{
Improving phase-transfer catalysis by enhancing non-covalent interactions
}

\author{
Iñigo Iribarren ${ }^{a}$ and Cristina Trujillo* $*^{a}$ \\ A wide variety of asymmetric transformations catalysed by chiral catalysts have been developed for the synthesis of valuable organic compounds in \\ the past several decades. Within asymmetric catalysis field, phase-transfer catalysis has been recognized as a powerful method for establishing \\ useful procedures for organic synthesis. In the present study intermolecular interactions between a well-known alkaloid quinine-derived phase \\ transfer catalyst and four different anions were characterised, analysing the competition between the pure ion-pair interaction and the \\ intermolecular hydrogen bond established upon complexation. Finally, the energy profile corresponding to the enantioselective conjugate \\ cyanation of a $\alpha, \beta$-unsaturated ketone, under the presence of two different catalysts were performed.
}

\section{Introduction}

During the past few decades, there has been an impressive interest in organocatalytic processes; the recovery and reuse of the catalyst is highly desirable from both economic and environmental standpoints. Due to the absence of transition metals, organocatalytic methods are attractive for the preparation of pharmaceutical compounds where levels of certain metal-ion contamination are tightly controlled.

Phase-transfer catalysts (PTCs), since Starks firstly introduced the term in $1971,{ }^{1}$ have been widely applied in organic reactions in different immiscible phases. Asymmetric PTCs based on the use of a variety of structurally well-defined chiral motifs have also attracted chemists' attentions, and have since been recognized as a versatile strategy for preparing chiral functional molecules. As a result, many novel organic transformations have been achieved with highly enantioselectivities. ${ }^{2,3}$

Several catalyst classes have been developed based on the Cinchona alkaloid and chiral binaphthyls have emerged among the most successful examples, including the quaternary -onium salts ${ }^{2,4-9}$ and applied successfully to highly enantioselective transformations.

Non-covalent interactions are of utmost importance across many different scientific domains from materials science to drug design. There is a diversity of non-covalent interactions, but the introduction of strong, directional hydrogen bonds ${ }^{10-12}$ in particular, are known to be crucial in the success of organocatalysed reactions It is well-known that the anion of the PTCs is usually situated proximally to the positively-charged nitrogen or phosphorous atom, establishing a strict (/tight) ion pairing interaction. ${ }^{13,}{ }^{14}$ By introducing secondary interactions, as in recent studies, ion pairing has found wide application in PTC-promoted asymmetric reactions ${ }^{3}$ (Figure 1 left). In bifunctional PTCs, however, ion pairing processes compete with the establishment of an intermolecular hydrogen bond between the PTC counteranion and potential hydrogen bond donors.
While the field of asymmetric organocatalysis is currently growing exponentially, an understanding of the mechanistic details involved in most of these reactions has often lagged far behind the pace of catalyst development, which in return retards rational catalyst design. Therefore, continuous efforts should be made toward the design and development of new catalysts classes, as well as understanding existing relationships between the structure of the catalyst and its ability to transfer stereochemical information. ${ }^{14-20}$

Herein, we present a theoretical study of the different interactions that can be established between the well-known alkaloid quinine derived PTC (Figure 1, right) and four different anions of interest in organocatalysis: $\mathrm{Cl}^{-}, \mathrm{Br}^{-}, \mathrm{MeCO}_{2}^{-}$and $\mathrm{CN}^{-}$. An improved PTC derivative to the existing system was proposed in order to increase the enantioselectivity of a model reaction and therefore a theoretical studied of the energy profile was performed. The enantioselective conjugate cyanation of $\alpha, \beta$-unsaturated ketones catalysed by the catalyst of interest and the modified one were studied.

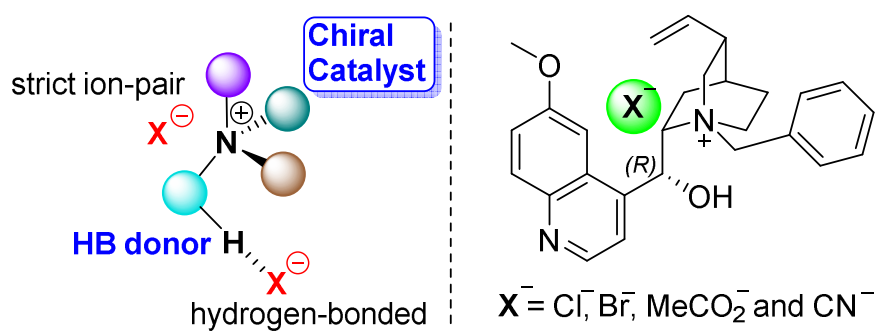

Figure 1. Structure of the different interacting systems analysed in the present study.

\section{Computational Details}

The structures of the complexes were optimized at M062X/aug-cc$\mathrm{pVDZ}^{21,22}$ computational level. Harmonic vibrational frequencies were computed at the same level used for the geometry optimizations in order to confirm that the stationary points are local minima. Calculations were performed using the Gaussian16 
software $^{23}$. Single point energies for lowest energy small basis set calculations were computed using M062X/aug-cc-pVTZ. Interaction energies $\left(\Delta G_{i}\right)$ were calculated as a difference of the energy of the optimised complex minus the energy of each monomer in their optimised geometry. The free energies reported in the document were obtained by adding the free energy correction from the small basis set calculations to the potential energy obtained from the high-level single-point energy calculations. Solvent effects (Toluene) were included in the optimization by means of a continuum method, the Solvation Model based on Density (SMD) approach $^{24}$ and the refined $\mathrm{SMD} 18^{25}$ version for $\mathrm{Br}$ atoms implemented in Gaussian16. The molecular electrostatic potential (MEP) of the isolated monomers were calculated on the electron density isosurface of $0.001 \mathrm{au}$. This isosurface was shown to resemble the van der Waals surface. ${ }^{26}$ These calculations were carried out with the Gaussian-16 software and the numerical results analysed using the Multiwfn ${ }^{27}$ and plotted using $\mathrm{Jmol}^{28}{ }^{28}$ The Atoms in Molecules (AIM) methodology ${ }^{29,30}$ was used to analyse the electron density of the systems with the AIMAll program. ${ }^{31}$ The Natural Bond Orbital (NBO) method ${ }^{32}$ was employed to evaluate atomic charges using the NBO-6 program, and to analyse charge-transfer interactions between occupied and unoccupied orbitals.

\section{Results and Discussion}

\section{Conformational Analysis of the Catalyst}

We began by exploring the low-energy chemical space of the catalyst by rotating three different parts of the catalyst plotted in Figure 2. The relative energies are summarised in Table S1.

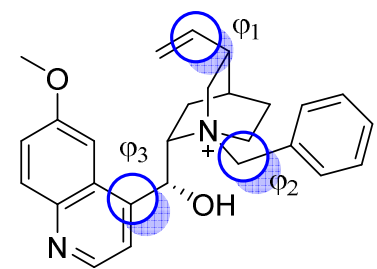

Figure 2. Different rotations taken into account for the conformational analysis.

From the conformational analysis 12 different conformers were obtained (Figure S1). The relative free energies range from 0.1 to $36.0 \mathrm{~kJ} \cdot \mathrm{mol}^{-1}$. Following the different conformations, it is clear that compared to catalyst conf_1 (no rotations made) when only $\varphi_{2}$ or $\varphi_{3}$ is rotated (conf_5 or conf_6, respectively Figure S1) the energy difference is $c a .14 \mathrm{~kJ} \cdot \mathrm{mol}^{-1}$, and therefore $c a .30 \mathrm{~kJ} \cdot \mathrm{mol}^{-1}$ is obtained when two dihedral angles are changed (conf_11 and conf_12, Figure S1). Rotations of the dihedral angle $\varphi_{1}$ do not provoke significant changes regarding the energy outcome.

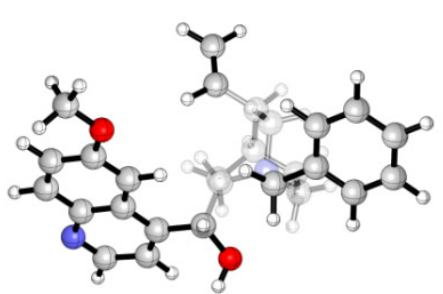

Figure 3. Lowest-energy optimised conformer for the catalyst under study, conf_1.

\section{Catalyst-Anion Interactions}

With the optimal conformations in hand, we began by calculating the molecular electrostatic potential (MEP) surface for the isolated catalyst in order to analyse the areas susceptible to anion interaction. Maxima values of the MEP on the 0.001 a.u. electron density isosurface were plotted in Figure 4 and summarised in Table 1.

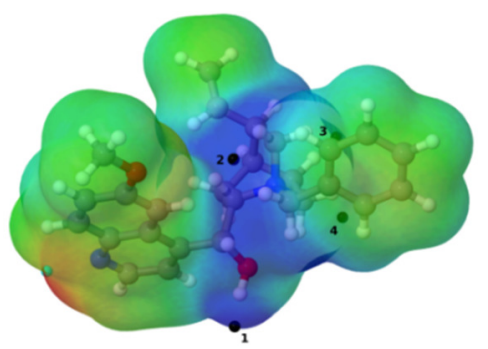

Figure 4. Molecular electrostatic potential on the 0.001 a.u electron density isosurface for the catalyst under study at the M062X/aug-cc-pVDZ computational level. Colour scheme range: $\operatorname{Red}(-0.015$ a.u.) to Blue $(+0.015$ a.u.).

Four MEP maxima values (black dots) were found within the catalyst $\left(\mathrm{V}_{\max }\right)$, one corresponding to the $\mathrm{OH}$ group and three localised around the quinuclidine ring, where the positive charge is placed (Figure 4). All the values are very close, but the most positive one corresponds to the $\mathrm{OH}$ group. Considering the previous results, it is clear that the hydrogen bond becomes a significant competitive interaction for the anion.

Table 1. Maxima $\left(\mathrm{V}_{\max }\right)$ values of the molecular electrostatic potential (in a.u.) on the 0.001 a.u. electron density isosurface for all the monomers (A) calculated at M062X/aug-cc-pVDZ level.

\begin{tabular}{lc}
\hline & $\mathbf{V}_{\max }$ \\
\hline $\mathbf{1}$ & 0.1779 \\
$\mathbf{2}$ & 0.1669 \\
$\mathbf{3}$ & 0.1500 \\
$\mathbf{4}$ & 0.1378 \\
\hline
\end{tabular}

In order to provide an insight of the different interactions that can be established with the catalyst a thorough computational study upon complexation were performed (Figure 1). The nomenclature chosen for the formed complexes is complex_X_N, where $\mathbf{X}$ refers the different anions involved $\left(\mathrm{X}=\mathrm{Cl}^{-}, \mathrm{Br}^{-}, \mathrm{MeCO}_{2}^{-}\right.$and $\left.\mathrm{CN}^{-}\right)$and $\mathbf{N}$ corresponds to the different maxima value found in the MEP of the catalyst, $(\mathbf{N}=\mathbf{1 - 5})$. Not in all cases the complex with $\mathrm{N}=4$ was found, 
and for both halogen anions an extra complex $\mathrm{N}=5$ was found. The optimised structures are shown in Figure S2. The interaction free energy values $\left(\Delta G_{\text {int }}\right)$ were gathered in Table 2 and they range from -74.5 to $-172.3 \mathrm{~kJ} \cdot \mathrm{mol}^{-1}$.

Regarding the different anions, the acetate anion complexes present the strongest interactions while the bromide ion complexes show the weakest interaction energy among of all them. It is clear that within the same anion-complex interaction, the one stablishing an intermolecular hydrogen bond exhibit the strongest interaction. If a comparison is made between the HB-bound complex (1) and the one exhibiting an strict ion-pair interaction, in which both charged atoms are closest (2), the former is in general more than $30 \mathrm{~kJ} \cdot \mathrm{mol}^{-1}$ more stable than the latter, even though the $\mathrm{X}^{\cdots \cdots} \mathrm{N}^{+}$distance is shorter.

Table 2. Interaction energies $\left(\Delta G, \mathrm{~kJ} \cdot \mathrm{mol}^{-1}\right), \mathrm{X} \cdots \mathrm{H}$ and $\mathrm{X} \cdots \mathrm{N}^{+}$intermolecular distances (Å) calculated at the M062X/aug-cc-pVTZ//aug-cc-pVDZ computational level.

\begin{tabular}{|c|c|c|c|}
\hline Complex & $\Delta \mathrm{G}_{\text {int }}$ & Dist. X"'H & Dist. $\mathrm{X}^{\prime} \mathrm{N}^{+}$ \\
\hline Complex_Cl_1 & -150.7 & 2.054 & 4.020 \\
\hline Complex_Cl_2 & -118.1 & - & 3.713 \\
\hline Complex_Cl_3 & -101.4 & - & 4.024 \\
\hline Complex_Cl_4 & - & - & - \\
\hline Complex_Cl_5 & -95.9 & - & 4.575 \\
\hline Complex_Br_1 & -139.6 & 2.259 & 4.217 \\
\hline Complex_Br_2 & -105.5 & - & 3.934 \\
\hline Complex_Br_3 & -92.4 & - & 4.255 \\
\hline Complex_Br_4 & -96.8 & - & 4.771 \\
\hline Complex_Br_5 & -86.9 & - & 4.758 \\
\hline Complex_MeCO ${ }_{2} 1$ & -172.3 & 1.491 & 4.729 \\
\hline Complex_MeCO ${ }_{2} 2$ & -133.6 & - & 3.410 \\
\hline Complex_MeCO ${ }_{2} 3$ & -107.0 & - & 3.591 \\
\hline Complex_MeCO 24 & - & - & - \\
\hline Complex_CN_1 & -142.8 & 1.684 & 3.810 \\
\hline Complex_NC_1 & -139.6 & 1.776 & 3.931 \\
\hline Complex_CN_2 & -107.5 & - & 3.461 \\
\hline Complex_CN_3 & -74.5 & - & 3.708 \\
\hline
\end{tabular}

Atoms in Molecules (QTAIM) analysis was carried out. The main electron density properties at the bond critical points (BCP) of the different HBs found in these complexes within the QTAIM analysis are summarized in Table S2. Molecular graphs for all the complexes were summarised in Table S1. The values of the electron density at the $B C P, \rho(B C P)$, found range from 0.079 to 0.004 a.u. Large $\rho(B C P)$ values indicate strong hydrogen bonds, while positive values of $\nabla^{2} \rho(B C P)$ are characteristic of close shell systems. From the molecular graphs and the analysis of the density at the BCPs, it is clear that the different anions can established either a strong intermolecular HB with the hydroxyl group of the catalyst $\left(\mathbf{O H}^{\prime \prime} \mathbf{X}\right)$ or mild hydrogen bonds with weaker hydrogen bond donors such as $\mathrm{CH}$ groups from the quinuclidine ring of the catalyst. The values both for $\rho(B C P)$ and $\nabla^{2} \rho(B C P)$ increase with the intermolecular hydrogen bond established between the anion and the $\mathrm{OH}$ group of the catalyst as seen in Table S2. Also, the strength of the most relevant intermolecular hydrogen bond $\left(\mathbf{O H}^{\prime} \mathbf{W}^{-} \mathbf{)}\right)$ is also dependent on the nature of the anion. It becomes weaker in the $\mathrm{MeCO}_{2}{ }^{-}>\mathrm{CN}^{-}$ $>\mathrm{Cl}^{-}>\mathrm{Br}^{-}$order, evidenced by the interaction energy, binding distance and electron density. That pattern is in complete agreement with the electronegative trend; greater electronegativity of the hydrogen bond acceptor will lead to an increase in hydrogenbond strength. Exponential relationships between the intermolecular distance with the anion and $\rho(B C P)$ in each $B C P$ were found (Figure 5).

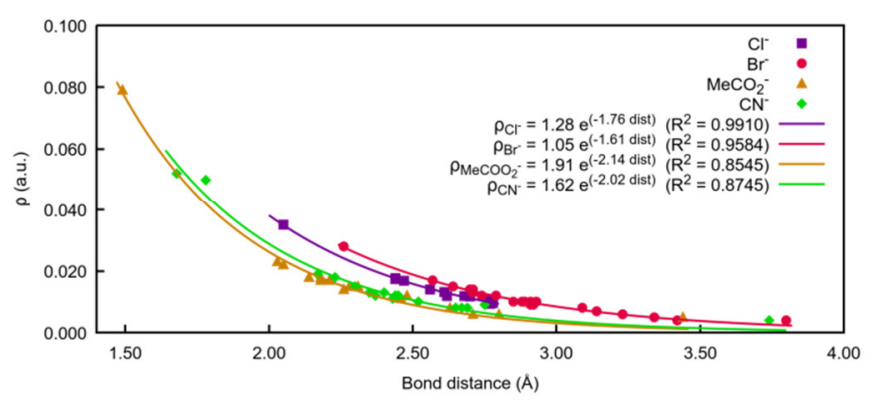

Figure 5. Exponential relationships between intermolecular distance catalyst and anion, in $\AA$, and $\rho(B C P)$.

In order to provide a visual description of the electron density overlap established in the intramolecular interactions, NonCovalent Interaction index (NCl) plots for the most representative examples (complex_X_1) were calculated and plotted (Figure 6). Besides, the 2D-NCl plots were depicted in Figure S4.
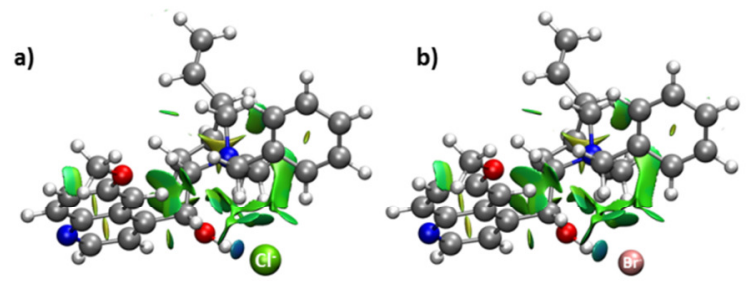

c)
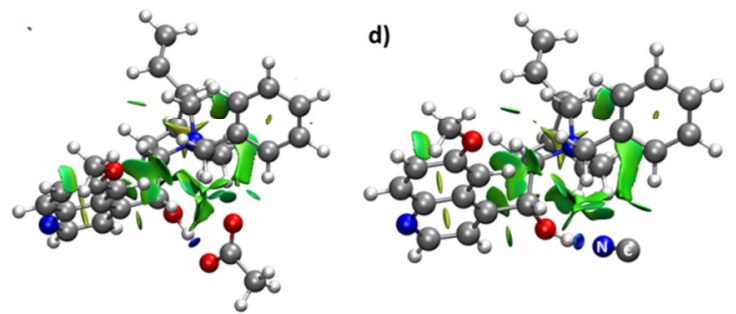

Figure 6. $\mathrm{NCl}$ plots of the interactions found for the most stable complex with $\mathrm{Cl}^{-}(\mathbf{a}), \mathrm{Br}^{-}$(b), $\mathrm{MeCO}_{2}{ }^{-}$(c) and $\mathrm{CN}^{-}(\mathbf{d})$. RDG isosurfaces provide a 3D representation of interaction regions. The sign of the second eigenvalue of the $\rho$ Hessian matrix is used to differentiate repulsive $\left(\lambda_{2}>0\right)$ from attractive $\left(\lambda_{2}<0\right)$ interactions.

In all the cases a green area corresponding to values of $\lambda_{2} \approx 0$ (weakly attractive) appears between the anions and the catalyst indicating the interactions taking place. In case of $\mathrm{MeCO}_{2}{ }^{-}$and $\mathbf{C N}^{-}$, two blue areas $\lambda_{2}<0$ (strongly attractive) are shown in coincidence with the position of the established hydrogen bond interaction. Those are consistent with the $\Delta \mathrm{G}_{\text {int }}$ values shown for both anion complexes. Also, $\mathrm{Cl}^{-}$complex presents small blue areas, weaker compare with the other two anions, but stronger than in bromide case. The calculated 2D-NCl plots show an intense peak corresponding to both anions, $\mathrm{MeCO}_{2}^{-}$and $\mathrm{CN}^{-}$, at more negative values of the $\lambda_{2}$.

Finally, an NBO analysis was used to identify and characterised intermolecular charge transfer between occupied molecular 
orbitals and empty ones upon complexation. In Table $\mathbf{S 5}$ the second order perturbation energies $E(2)$ present in the complexes found are reported. Among all the molecular interactions observed the largest contributions correspond to the donations from the $X^{-}$ electron lone pairs into the $\sigma^{*}$ antibonding orbital of the hydrogen bond donor $\left(\sigma^{*} \mathrm{O}-\mathrm{H}\right)$. In all the anion-hydrogen bonding established in the series the orbital interaction decrease following the interaction energy trend $\left(\mathrm{MeCO}_{2}^{-}>\mathrm{CN}^{-}>\mathrm{Cl}^{-}>\mathrm{Br}^{-}\right)$. The smallest contributions of the series come from the ion pair in which both ions present the larger interatomic distance.

\section{Conjugate Cyanation}

Having obtained a clearer understanding of the different interactions that can be established between the catalyst and the different anions, an application in order to improve the phase transfer catalysis of the cat1 was studied.

We began by studying the pathway of the enantioselective conjugate cyanation of $\alpha, \beta$-unsaturated ketone, $(E)$-Chalcone (1), under the presence of the catalyst previously studied, cat1, and then a theoretical modification of the catalyst was made, cat2 (Scheme 1). In order to improve the enantioselectivity of this reaction, an extra anchorage point was added.

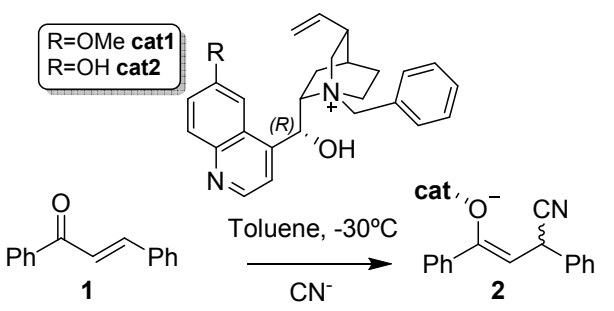

Scheme 1. Asymmetric conjugate cyanation of $(E)$-Chalcone.

Only the first step, addition of the $\mathrm{CN}^{-}$, was studied, since is the responsible of the stereochemistry of the global process, therefore the product of the energy profile is the cat-bound enolate (2). Besides, from the methodology perspective, due to convergence issues, a $6-31 \mathrm{~g}(\mathrm{~d})^{33,34}$ basis set was used to perform the energy profile, instead the previous used aug-cc-pVDZ basis set. To guarantee the homogeneity of the calculation along the project a benchmark between both bases set for the conformational study as well as for the catalyst-anion interactions study (Figures S2 and S3), obtaining, in general, the same trend.

The free energy profile for the formation of both enantiomers of cat1-bound enolate product (2) (Figure 7) indicates that the barrier corresponding for the $\mathrm{C}-\mathrm{C}$ bond formation is very similar for both pathways, around $80 \mathrm{~kJ} \cdot \mathrm{mol}^{-1}$ for both pathways $\left(\Delta \Delta \mathrm{G}=0.6 \mathrm{~kJ} \cdot \mathrm{mol}^{-}\right.$ $\left.{ }^{1}\right)$. It is clear from the energy profile that no sterical issues are presented by the two TSs ( $R$ and $S$ ) and therefore both are energetically accessible by the cyanide.

Regarding the cat-enolate complexes (2), they present similar energies to the pre-TS assembly, being the corresponding enantiomer $\mathrm{S}$ slightly more stable that the $R$ one.

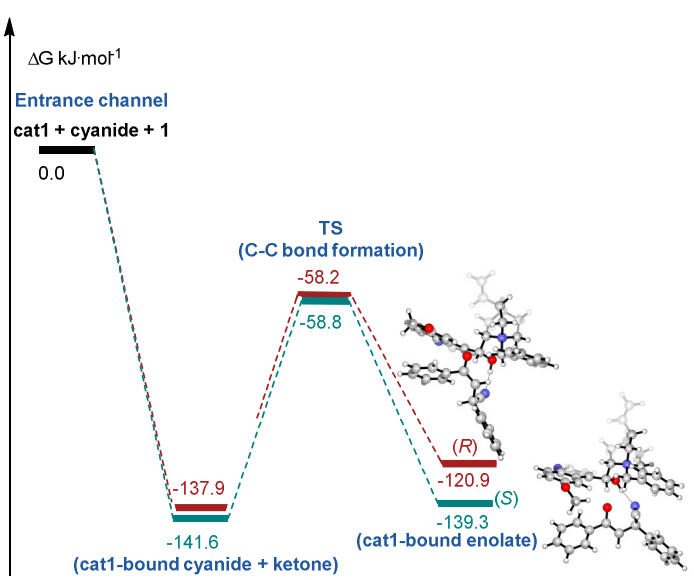

Figure 7. Free-energy profiles for the reaction under the presence of cat1.

When the OMe group is replaced by a hydrogen bond donor group, $\mathrm{OH}$, a second anchoring point is added, forcing the intermolecular hydrogen bond between the two moieties, new hydroxyl group of cat2 and the oxygen of the $(E)$-Chalcone. The free-energy profile study was performed (Figure 8), showing that the energetic barrier between the two enantiomers had increased up to $12 \mathrm{~kJ} \cdot \mathrm{mol}^{-1}$.

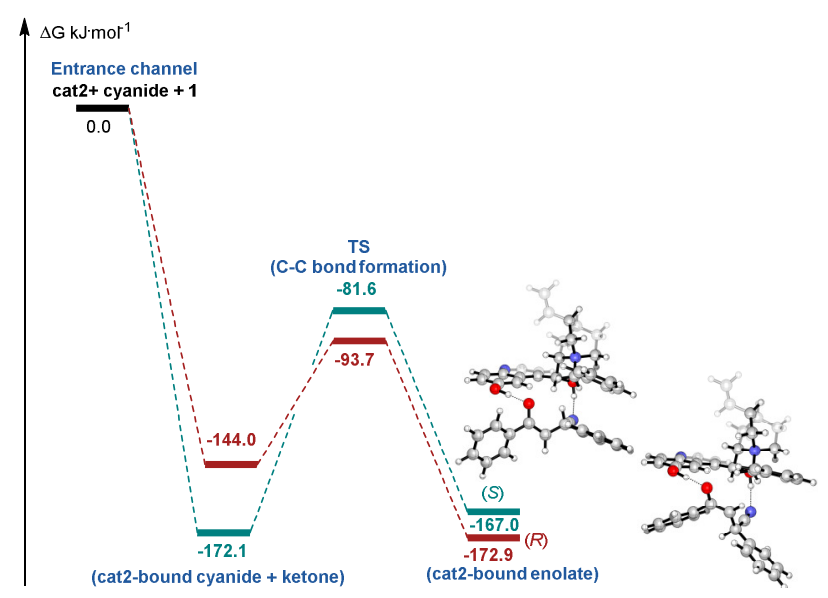

Figure 8. Free-energy profiles the reaction under the presence of cat2.

To probe the origins of the observed asymmetric induction, we examined the transition states associated with the key stereocentre forming addition step for both major enantiomers (Figure 9). The binding mode is analogous for both TSs.

Inspection of the calculated transition state leading to the major antipode allows an appreciation of why the simple modification, 6'hydroxylation, was superior in this process and also sheds light on the catalyst-substrate interactions which render aromatic aldehydederived imines considerably more amenable to highly enantioselective cycloaddition than aliphatic analogues. Cat2 forms a discernible non-covalent network identified by QTAIM calculations (see ESI) in the transition state. This, together with the binding of the $\mathrm{O}-\mathrm{H}$ proton to one of the carbonyl group, aligns the electrophile. 

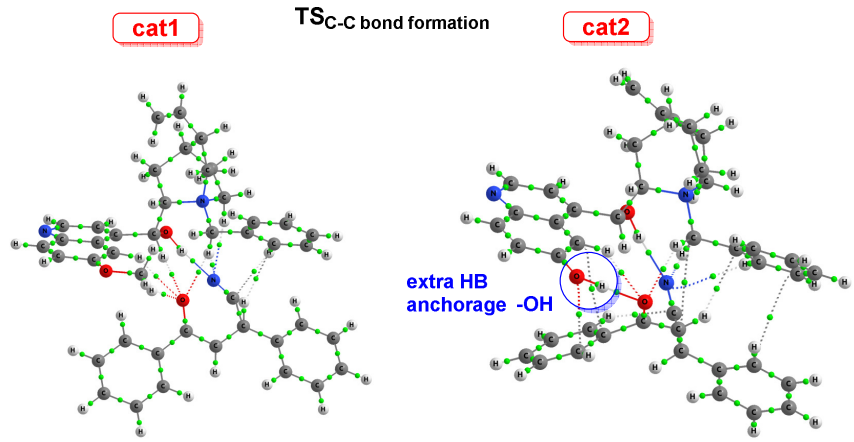

Figure 9. Optimised geometries for both TSs under the presence of cat1 (left) and cat2 (right) for the major enantiomer in each reaction ( $S$ and $R$ respectively).

The calculated ee is $13 \%$ when the reaction goes under the presence of cat1 while for cat 2 increases up to $99 \%$.

\section{Conclusions}

A theoretical study of the different interactions that can be established between the well-known alkaloid quinine -derived PTC and four different anions of interest in organocatalysis: $\mathrm{Cl}^{-}, \mathrm{Br}^{-}$, $\mathrm{MeCO}_{2}{ }^{-}$and $\mathrm{CN}^{-}$were performed. The competition between the ion pairing processes with the establishment of an intermolecular hydrogen bond between the PTC counteranion and potential hydrogen bond donors was studied.

It was found that the acetate anion complexes exhibit the strongest interactions while the bromide ion complexes show the weakest interaction energy among of all them, therefore, the one stablishing an intermolecular hydrogen bond present the strongest interaction. The complex exhibiting an strict ion-pair interaction, in which both charged atoms are closest (2) (shortest $\mathrm{X}^{-\cdots} \mathrm{N}^{+}$distance) is $30 \mathrm{~kJ} \cdot \mathrm{mol}^{-1}$ less stable than the HB-bound complex (1).

The free energy profile corresponding to the enantioselective conjugate cyanation of $\alpha, \beta$-unsaturated ketone, $(E)$-Chalcone, under the presence of the catalyst cat 1 was performed. The barrier for both enantiomers of cat1-bound enolate product indicates that the $\mathrm{C}-\mathrm{C}$ bond formation is very similar for both pathways, $\left(\Delta \Delta \mathrm{G}=0.6 \mathrm{~kJ} \cdot \mathrm{mol}^{-1}\right)$. From the energy profile both enantiomers ( $R$ and $S$ ) are energetically accessible by the cyanide, obtaining a theoretical ee value of $13 \%$.

In order to improve the enantioselectivity of this reaction, an extra anchorage point was added, cat2. The corresponding free energy profile was studied.The calculated $e e$ when the reaction goes under the presence of cat 2 increases up to $99 \%$.

\section{Conflicts of interest}

There are no conflicts to declare.

\section{Acknowledgements}

This publication has emanated from research supported by Science Foundation Ireland (SFI 18/SIRG/5517). We are grateful to the Irish Centre for High-End Computing (ICHEC) for the provision of computational facilities. We are indebted to Dr. Goar Sánchez from ICHEC for the AIM graphical outputs and Prof. Ibon Alkorta for the NBO-6 software. We would like to thank Prof. Stephen Connon for all useful discussions, ideas and his continuous support.

\section{Notes and references}

C. M. Starks, J. Am. Chem. Soc., 1971, 93, 195-199.

S. Shirakawa and K. Maruoka, Angew Chem Int Edit, 2013, 52, 4312-4348.

3. H. Wang, Catalysts, 2019, 9, 244.

4. T. Ooi and K. Maruoka, Angew Chem Int Edit, 2007, 46, 4222-4266.

5. J. Novacek and M. Waser, Eur. J. Org. Chem., 2013, 2013, 637-648.

6. S. Kaneko, Y. Kumatabara and S. Shirakawa, Org. Biomol. Chem., 2016, 14, 5367-5376.

7. S. Liu, Y. Kumatabara and S. Shirakawa, Green Chemistry, 2016, 18, 331-341.

8. A. Golandaj, A. Ahmad and D. Ramjugernath, Adv. Synth. Catal., 2017, 359, 3676-3706.

9. J. Schörgenhumer, M. Tiffner and M. Waser, Beilstein J. Org. Chem., 2017, 13, 1753-1769.

10. I. Alkorta, I. Rozas and J. Elguero, Chem. Soc. Rev., 1998, 27, 163-170.

11. P. A. Frey, Magn. Reson. Chem., 2001, 39, S190-S198.

12. M. Meot-Ner, Chem. Rev., 2005, 105, 213-284.

13. K. Brak and E. N. Jacobsen, Angew Chem Int Edit, 2013, 52, 534-561.

14. G. P. Petrova, H.-B. Li, K. Maruoka and K. Morokuma, J. Phys. Chem. B, 2014, 118, 5154-5167.

15. C. Trujillo, I. Rozas, A. Botte and S. J. Connon, Chem. Commun., 2017, 53, 8874-8877.

16. C. Trujillo, M. Litvajova, S. A. Cronin, R. Craig and S. J. Connon, ChemCatChem, 2019, 11, 3776-3780.

17. B. Lockett-Walters, C. Trujillo, B. Twamley and S. Connon, Chem. Commun., 2019, 55, 11283-11286.

18. A. G. Collar, C. Trujillo, B. Lockett-Walters, B. Twamley and S. J. Connon, Chem. Eur. J., 2019, 25, 7275-7279.

19. Y.-h. Lam, M. N. Grayson, M. C. Holland, A. Simon and K. N. Houk, Acc. Chem. Res., 2016, 49, 750-762.

20. P. H.-Y. Cheong, C. Y. Legault, J. M. Um, N. Çelebi-Ölçüm and K. N. Houk, Chem. Rev., 2011, 111, 5042-5137.

21. D. E. Woon and T. H. Dunning, J. Chem. Phys., 1995, 103, 4572-4585.

22. T. H. Dunning, J. Chem. Phys., 1989, 90, 1007-1023.

23. M. J. Frisch, G. W. Trucks, H. B. Schlegel, G. E. Scuseria, M. A. Robb, J. R. Cheeseman, G. Scalmani, V. Barone, G. A. Petersson, H. Nakatsuji, X. Li, M. Caricato, A. V. Marenich, J. Bloino, B. G. Janesko, R. Gomperts, B. Mennucci, H. P. Hratchian, J. V. Ortiz, A. F. Izmaylov, J. L. Sonnenberg, Williams, F. Ding, F. Lipparini, F. Egidi, J. Goings, B. Peng, A. Petrone, T. Henderson, D. Ranasinghe, V. G. Zakrzewski, J. Gao, N. Rega, G. Zheng, W. Liang, M. Hada, M. Ehara, K. Toyota, R. Fukuda, J. Hasegawa, M. Ishida, T. Nakajima, Y. Honda, O. Kitao, H. Nakai, T. Vreven, K. Throssell, J. A. Montgomery Jr., J. E. Peralta, F. Ogliaro, M. J. Bearpark, J. J. Heyd, E. N. Brothers, K. N. Kudin, V. N. 
Staroverov, T. A. Keith, R. Kobayashi, J. Normand, K. Raghavachari, A. P. Rendell, J. C. Burant, S. S. Iyengar, J. Tomasi, M. Cossi, J. M. Millam, M. Klene, C. Adamo, R. Cammi, J. W. Ochterski, R. L. Martin, K. Morokuma, O. Farkas, J. B. Foresman and D. J. Fox, Journal, 2016.

24. A. V. Marenich, C. J. Cramer and D. G. Truhlar, J. Phys. Chem. B, 2009, 113, 6378-6396.

25. E. Engelage, N. Schulz, F. Heinen, S. M. Huber, D. G. Truhlar and C. J. Cramer, Chem. Eur. J., 2018, 24, 1598315987.

26. R. F. W. Bader, M. T. Carroll, J. R. Cheeseman and C. Chang, J. Am. Chem. Soc., 1987, 109, 7968-7979.

27. T. Lu and F. Chen, J. Comput. Chem., 2012, 33, 580-592.

28. Journal.

29. R. F. W. Bader, Atoms in Molecules: A Quantum Theory, Clarendon Press, Oxford, 1990.

30. P. L. A. Popelier, Atoms In Molecules. An introduction, Prentice Hall, Harlow, England, 2000.

31. T. A. Keith, Journal, 2011, TK Gristmill Software,(aim.tkgristmill.com).

32. A. E. Reed, L. A. Curtiss and F. Weinhold, Chem. Rev., $1988,88,899-926$.

33. G. A. Petersson, A. Bennett, T. G. Tensfeldt, M. A. AlLaham, W. A. Shirley and J. Mantzaris, The Journal of Chemical Physics, 1988, 89, 2193-2218.

34. G. A. Petersson and M. A. Al-Laham, The Journal of Chemical Physics, 1991, 94, 6081-6090. 